\title{
An Optimized User Selection Method for Cooperative Diversity Systems
}

\author{
Diomidis S. Michalopoulos*§, George K. Karagiannidis*, Theodoros A. Tsiftsis ${ }^{\dagger}$ and Ranjan K. Mallik \\ * Telecommunications Division, Department of Electrical \& Computer Engineering, Aristotle University of Thessaloniki, GR-54124, Thessaloniki, Greece \\ Email:\{dmixalo, geokarag\}@auth.gr

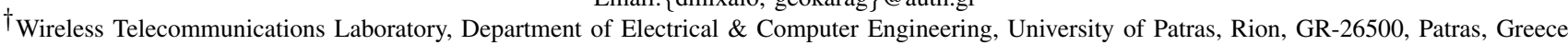 \\ Email: tsiftsis@ee.upatras.gr \\ ${ }^{\ddagger}$ Department of Electrical Engineering, Indian Institute of Technology - Delhi, Haus Khas, New Delhi 110016, India (e-mail: rkmallik@ee.iitd.ernet.in)

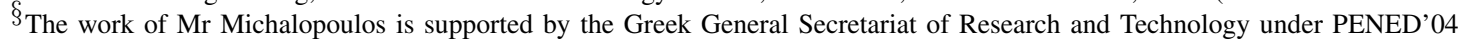

\begin{abstract}
Multi-user cooperative diversity is a recent technique promising great improvement of the performance of wireless communication systems operating in fading environments. Based on combinatorial optimization theory and specifically on the so-called knapsack problem, this paper presents a method of optimizing the selection among the potential cooperating users, when amplify-and-forward relays are used. In particular, two optimization problems are studied: the error probability minimization subject to total energy consumption constraints, and the dual one, the energy consumption minimization under error performance constraints. Depending on the frequency of repeating this selection, the above problems are categorized into shortterm and long-term node selection. Numerical examples verify the expected knapsack scheme's advantage of adapting the number of cooperating users, depending on the desired performanceconsumption tradeoff. Moreover, long-term node selection seems to lead to similar error or consumption performance compared to the short-term one, despite its simplicity.
\end{abstract}

\section{INTRODUCTION}

In the last few years, a new concept that is being actively studied in multihop-augmented networks is multi-user cooperative diversity, where several terminals form a kind of coalition to assist each other with the transmission of their messages. In general, cooperative relaying systems have a source node multicasting a message to a number of cooperative relays, which in turn resend a processed version to the intended destination node. The destination node combines the signal received from the relays, taking into account the source's original signal [1]- [4].

The main contribution of this paper is the proposal of a novel node-selection strategy for multi-user cooperative diversity systems, according to which only a subset of the set of available users is activated, in order to achieve the optimal balance between error performance and total consumed energy. More specifically, two variations of this problem are introduced: the end-to-end error performance optimization under total power constraints, and the dual one, the minimization of the total consumed energy provided that the end-to-end error probability will not exceed a predefined threshold. This is accomplished by utilizing the general concept of optimizing the selection among the elements of a given set under specific constraints, which was first introduced in combinatorial optimization theory: Given an item set, and assuming that each item is characterized by a unique pair of profit and weight values, the subset that maximizes the profit summation provided that the weight summation does not exceed a maximum value needs to be distinguished. These problems are widely known as knapsack problems [5].

In this work, dual-hop amplify-and-forward cooperative diversity is assumed, where a number of individual relays amplify and retransmit the signal received from the source node to the destination one. The structure of the studied model is further analyzed in Section II; in Section III, the knapsack application on cooperative diversity systems is presented. In such applications, the gain and the energy each relay consumes depends usually only on the fading state of its input channel, in order to limit the output power of the relay. Moreover, assuming maximal ratio combining (MRC) at the receiver, the instantaneous ${ }^{1}$ overall signal to noise ratio (SNR) is the sum of the end-to-end SNRs corresponding to each separate branch. These end-to-end SNRs, reflect the branches' ability to contribute to the total performance enhancement, and are also determined by a combination of the fading state of the channels in the input and the output of the corresponding relays. We thus realize that two scalar metrics can be attributed to each branch, depending only on the fading conditions corresponding to it, and characterizing its contribution to the end-to-end performance and its energy consumption respectively. Then, the error performance optimization problem under total energy consumption constraints, and the dual problem of minimizing the energy consumption provided that the received SNR is not arbitrarily small, can be reduced to knapsack ones. This requires that the selection is repeated on a frequent basis, at a rate that ensures constant fading characteristics in every selection interval. Next, this type of selection is referred as short-term node selection (STNS), and is further analyzed in Section IV.

In Section V, a solution that tackles the above problems in an average sense is presented. More specifically, since all channels are considered ergodic, the metrics that are attributed to the corresponding relays are determined by their fading

\footnotetext{
${ }^{1}$ In the following, the term instantaneous will be used in a loose sense, describing the time interval in which the fading state can be considered constant.
} 
statistics, and the selection problems are again reduced to knapsack ones, optimizing the system in a long-term point of view. For this reason, this method is referred as long-term node selection (LTNS). Finally, in Section VI a set of numerical examples are discussed.

\section{System AND ChanNEL MODEL}

We consider a system with a source node $S$, communicating with a destination node $D$, with the aid of $L$ other cooperating nodes which act as non-regenerative relays, denoted by $R_{j}$, $j=1, \ldots, L$. Let $b_{j}$ stand for the system branch corresponding to the relay $R_{j}$. Each transmission period $T$ is divided into two slots: in the first slot, node $S$ communicates with the relays and the destination terminal, while in the second slot, only the relays communicate with node $D$. The above transmission protocol was originally proposed in [6].

Let us assume that the system is operating over independent but not necessarily identically distributed Nakagami- $m$ fading channels, node $S$ transmits with instantaneous power normalized to unity and the gain $G_{j}$ of $R_{j}$ is set to counterbalance the signal degradation in the hop $S-R_{j}$ in order to limit its output power [3] i.e.,

$$
G_{j}^{2}(t)=\frac{P_{j, \text { out }}}{a_{S j}^{2}(t)+N_{0}},
$$

where $P_{j, \text { out }}$ is the relay's transmission power, and $a_{S j}(t)$, $N_{0}$ are the fading amplitude and the additive white Gaussian noise (AWGN) power of the $S-R_{j}$ channel, respectively. In such case, the instantaneous $\operatorname{SNR} \gamma_{j}$, of the $b_{j}$ branch is

$$
\gamma_{j}=\frac{\gamma_{S j} \gamma_{D j}}{\gamma_{S j}+\gamma_{D j}+1}
$$

where $\gamma_{S j}=a_{S j}^{2} / N_{0}, \gamma_{D j}=P_{j, \text { out }} a_{D j}^{2} / N_{0}$ are the instantaneous SNR of the hop $S-R_{j}$ and $R_{j}-D$, respectively. Assuming that $l$ nodes are operating during any time interval $T$, the overall instantaneous SNR at the output of the MRC at the destination terminal during this interval can be written as

$$
\gamma_{e n d}=\gamma_{0}+\sum_{j=1}^{l} \frac{\gamma_{S j} \gamma_{D j}}{\gamma_{S j}+\gamma_{D j}+1}=\sum_{j=0}^{l} \gamma_{j}
$$

where $\gamma_{0}$ is the instantaneous SNR of the direct $S-D$ channel. The system branch corresponding to the $S-D$ channel is denoted by $b_{0}$.

\section{The KnApsack Problem And its Application on COOPERATIVE DIVERSITY SYSTEMS}

\section{A. The Knapsack Problem}

1) General Description: The well-known zero-one knapsack problem is defined as follows:

Given an item set $\mathcal{N}$, consisting of $n$ items with profits $p_{j}>0$ and weights $w_{j}>0, j=1, \ldots, n$, and given the capacity value $C_{\max }$, select the subset of $\mathcal{N}$ such that the total profit of the selected items is maximized and the total weight does not exceed $C_{\max }$. In other words,

$$
\begin{gathered}
\text { maximize } \sum_{j=1}^{n} p_{j} x_{j} \\
\text { subject to } \sum_{j=1}^{n} w_{j} x_{j} \leq C_{\max } \\
x_{j} \in\{0,1\}, \quad j=1, \ldots, n .
\end{gathered}
$$

A variation of this problem can be expressed by minimizing, instead of maximizing, the profit summation, under the constraint that the total weight is greater than or equal to a given value $C_{\min }$, i.e.,

$$
\begin{gathered}
\text { minimize } \sum_{j=1}^{n} p_{j} y_{j} \\
\text { subject to } \sum_{j=1}^{n} w_{j} y_{j} \geq C_{\min } \\
y_{j} \in\{0,1\}, \quad j=1, \ldots, n .
\end{gathered}
$$

In what follows, we refer to the problems having the form of (4) as traditional knapsack problems, and to the ones with the form of (5) as minimization knapsack problems. Observing these two versions, it is clear that although their aim is completely different, their original concept is identical, stating that a separation has to be made among the items, in terms of their profits and weights combination. For this reason, both these kind of problems are referred as knapsack ones, and the algorithms that tackle them are similar [5].

2) Efficient Knapsack Algorithms: There is very strong theoretical evidence that for the knapsack problem no polynomial time algorithm exists for computing its optimal solution [5]. Within the general approach of knapsack problems, many algorithms are known that come close to an optimal solution, so as the difference between the values of the optimal and the approximate solution is small. Thus, due to the fact that algorithms with high complexity are undesirable for our system, we focus on algorithms computing a suboptimal solution in a relatively short amount of time. One efficient suboptimal algorithm is the well-known Greedy one, which operates as follows:

Algorithm 1 (Traditional Knapsack problem): "For every item $j \in \mathcal{N}$, denote with $e_{j}$ the profit to weight ratio, which is also called the efficiency of this item, e.g.,

$$
e_{j}:=\frac{p_{j}}{w_{j}}
$$

Sort the items in decreasing order of efficiency, and then start with an empty knapsack and simply go through the items in this order adding every item under consideration into the knapsack, if the capacity constraint is not violated thereby. "

Algorithm 2 (Minimization Knapsack problem): "For every item $j \in \mathcal{N}$, denote with $e_{j}$ the weight to profit ratio e.g.,

$$
e_{j}:=\frac{w_{j}}{p_{j}}
$$


Sort the items in decreasing order of efficiency, and then start with an empty knapsack and simply go through the items in this order, adding every item into the knapsack unless $\sum_{j=1}^{L} w_{j} x_{j} \geq C_{\min }$ is satisfied."

\section{B. Utilizing the Knapsack Approach in Cooperative Diversity Systems}

In cooperative diversity systems, the set $\mathcal{N}$ can be regarded as the set of all available system branches, including $b_{0}$. This branch-set is denoted by $\mathcal{R}$, e.g.,

$$
\mathcal{R}=\left\{b_{0}, b_{1}, \ldots, b_{L}\right\} .
$$

The problems that arise now are which branches to activate (if possible) during specific time intervals, in order to optimize system end-to-end error performance under average power constraints, or to minimize the total consumed energy provided that the error probability will not exceed a given threshold. In the following sections, we prove that these problems can be formulated as traditional and minimization knapsack problems respectively, by substituting the parameters of (4) and (5) with the appropriate system quantities.

1) The Branch-Set $\mathcal{R}$ : In general, $\mathcal{R}$ represents the set of all the available for cooperation branches; a node is considered available if it satisfies a number of relaying conditions, which reflect its possibility and willingness to act as a relay. These conditions may depend generally on the node's energy deposit, on its ability to forward the information in an adequate rate, on the synchronization cost its activation may entail etc. However, further analysis of these relaying conditions, followed by the question of under which circumstances relaying is a worthy technique, are beyond the scope of this paper, and are left for future work.

2) Cooperative Diversity's Special Knapsack Features: Considering that, in general, the coefficients $p_{j}, w_{j}$ correspond to a performance and energy consumption metric respectively, the following are the main points in which the branch selection in cooperative diversity systems differs from the typical knapsack applications:

- In cooperative diversity systems, the total weight capacity does not represent a strictly fixed value with a physical sense, as it occurs in the majority of knapsack applications. On the contrary, it reflects the concept of limiting the total number of cooperating nodes and the extra energy consumed to only a single user's avail.

- The amount of time needed for the algorithm computation in cooperative diversity systems is very important, since it has to be small enough in order not to cause any significant delay in packet transmission.

- In cooperative diversity systems, the direct branch $b_{0}$ is always activated since this does not entail any extra consumed energy.

Thus, it is evident that the Greedy algorithm tackles, in a relatively adequate amount of time, the problem of selecting the branches with optimal compromise between contribution to the total performance and energy consumption, provided that the total energy consumed for a single user's communication is limited. For this reason, Algorithm 1 and Algorithm 2, slightly modified in order to always include $b_{0}$, are regarded as a very good solution in the branch selection problem.

\section{Short-Term Node Selection (STNS)}

Let $T_{c}$ be the channel coherence time. Assume that each relay $R_{j}$ has full knowledge of the CSI of the $S-R_{j}$ and $R_{j}$ $D$ hop, and that the relays are capable of informing the source node about their channel state conditions, in a time much shorter than $T_{c}$. In slow fading environments, these conditions do not significantly change within time intervals approximately equal to $T_{c}$. Therefore, the transmitter can decide every $T_{c}$ which of the relays will operate for the next interval $T_{c}$, in order to minimize the outage probability $P_{o}^{2}$ under total power constraints, or to minimize the total consumed energy provided that $P_{o}$ will not exceed a predefined value, for this interval. Hence, STNS implies that this selection will be repeated frequently enough, ensuring that the fading conditions of each branch will remain constant until the next selection. This requires advanced cooperation protocols and efficient selection algorithms, with relatively small computation time compared to the duration of the transmitted symbols.

\section{A. $P_{o}$ Minimization Under Energy Consumption Constraints}

Since $\gamma_{e n d}$ at the output of the MRC at terminal $D$ remains constant during $T_{c}$, the node-selection that minimizes $P_{o}$ is the selection that maximizes $\gamma_{e n d}$, or equivalently the selection that maximizes $\sum_{j=1}^{l} \gamma_{j}$, for this interval. The energy $\mathcal{E}_{j}$ that the relay $R_{j}$ consumes per unit time equals to

$$
\mathcal{E}_{j}=\left(P_{j, \text { out }}-P_{j, \text { in }}\right)^{+},
$$

where $P_{j, i n}$ is the signal power at the input of $R_{j}$ and $(\cdot)^{+}=$ $\max (\cdot, 0)$. Eq. (9) reflects the fact that, in the case when $G_{j}>$ 1 the consumed power equals the difference between $P_{j, \text { out }}$ and $P_{j, i n}$, whereas in the case when $G_{j} \leq 1$ the consumed power is zero, since such attenuation can be achieved using a simple voltage divider. In each case, however, the energy consumed for any signal processing at the relays is considered negligible. Thus, $\mathcal{E}_{j}$ can be expressed as

$$
\mathcal{E}_{j}=\left(\left(1-\frac{1}{G_{j}^{2}}\right) P_{j, \text { out }}\right)^{+},
$$

or by using (1) as

$$
\mathcal{E}_{j}=\left(P_{j, \text { out }}-a_{S j}^{2}-N_{0}\right)^{+} .
$$

Considering the above, the node-selection problem can be formulated as a traditional knapsack one, i.e.,

$$
\begin{aligned}
& \text { maximize } \sum_{j=0}^{L} \gamma_{j} x_{j} \\
& \text { subject to } \sum_{j=1}^{L} \mathcal{E}_{j} x_{j} \leq C_{\max } \\
& x_{j} \in\{0,1\}, \quad j=0,1, \ldots, L .
\end{aligned}
$$

\footnotetext{
${ }^{2}$ As outage probability $P_{o}$ we define the probability that the instantaneous bit error probability exceeds a specified value [7, p. 5].
} 
where $\gamma_{j}$ and $\mathcal{E}_{j}$ are given in (2) and (11), respectively. The value of $C_{\max }$ in the last equation represents an instantaneous consumed power constraint, which can be adjusted in order to achieve the desirable tradeoff between performance and power consumption. Recall that the $L$ branches used in (12) are those defined by the set $\mathcal{R}$.

\section{B. Total Energy Consumption Minimization Under $P_{o}$ Con- straints}

Another application of the knapsack theory into cooperative diversity systems is the minimization of the total consumed energy, provided that the outage probability $P_{o}$ will not exceed a predefined value $\delta$ (if possible ${ }^{3}$ ), or equivalently that the $\gamma_{\text {end }}$ is lower-bounded, for a time interval $T_{c}$. The problem thus reduces to a minimization knapsack one, having the form of

$$
\begin{gathered}
\text { minimize } \sum_{j=1}^{L} \mathcal{E}_{j} x_{j} \\
\text { subject to } \sum_{j=0}^{L} \gamma_{j} x_{j} \geq C_{\min } \\
x_{j} \in\{0,1\}, \quad j=0,1, \ldots, L .
\end{gathered}
$$

As in (12), the value of $C_{\min }$ in (13) can be seen as a measure of the upper bound $\delta$ of $P_{o}$, taking into account the desirable performance-power consumption tradeoff. In a BPSK application, for example, $C_{\min }$ is related with $\delta$ through

$$
\operatorname{erfc}\left(C_{\min }\right)=2 \delta
$$

with $\operatorname{erfc}(\cdot)$ being the well-known complementary error function.

From a long-term point of view, in both these systems, after a large number of selections the average bit error probability (ABEP) is either minimized or upper-bounded by a constant $C_{1}$, while the average total consumed energy is either upperbounded by a constant $C_{2}$ or minimized, respectively. The values $C_{1}$ and $C_{2}$ are also the upper bounds of the $P_{o}$ and the consumed energy in every time interval $T_{c}$. We thus realize that STNS sets a tight constraint on the final ABEP and total energy consumption, keeping them limited in each interval $T_{c}$. Consequently, this type of selection does not exploit any possible knowledge on channel statistics, which vary much more slowly than the instantaneous channel state and can be effectively estimated in practical applications [8]- [9].

\section{Long-Term Node Selection (LTNS)}

Let us assume that the transmitter has full knowledge of the fading statistics corresponding to each of the ergodic channels $S-D, S-R_{j}$ and $R_{j}-D, j=1, \ldots, L$. The two optimization problems described above can now be implemented in an average sense, formulating equivalent traditional

\footnotetext{
${ }^{3}$ Obviously, in cases when the activation of all the available relays results in a $P_{o}$ which is greater than the predefined threshold the constraint cannot be satisfied.
}

and minimization knapsack problems, with different optimization and constraint parameters. Their main advantages are less frequent computation and less complicated cooperation protocols, which obviously makes them easier to implement in most practical cases. In fact, LTNS is repeated in a rate ensuring that the fading statistics remain constant during each selection interval.

\section{A. ABEP Minimization Under Energy Consumption Con- straints}

Since the total energy consumption is constrained, the weight $w_{j}$ of the branch $b_{j}, j=1, \ldots, L$, is the energy consumed by the corresponding relay, i.e.,

$$
w_{j}=\left\{\begin{array}{cc}
E\left[\mathcal{E}_{j}\right], & j=1, \ldots, L \\
0, & j=0
\end{array}\right.
$$

Assuming Nakagami- $m$ fading and unitary power transmitted by $S, E\left[\mathcal{E}_{j}\right]$ can be approximated in the medium and high SNR regime using (11) as

$$
\begin{gathered}
E\left[\mathcal{E}_{j}\right] \approx \int_{0}^{1}(1-x) \frac{m_{S j}^{m_{S j}} x^{m_{S j}-1}}{\Omega_{S j}^{m_{S j}} \Gamma\left(m_{S j}\right)} \exp \left(-\frac{m_{S j} x}{\Omega_{S j}}\right) d x \\
=1-\Omega_{S j}+\frac{\left(m_{S j}+1\right) \Omega_{S j} \Gamma\left(m_{S j}+1, \frac{m_{S j}}{\Omega_{S j}}\right)}{\Gamma\left(m_{S j}+2\right)} \\
-\frac{m_{S j}\left(m_{S j}+1\right) \Gamma\left(m_{S j}, \frac{m_{S j}}{\Omega_{S j}}\right)}{\Gamma\left(m_{S j}+2\right)}
\end{gathered}
$$

where $\Omega_{S j}=E\left[a_{S j}^{2}\right]$ and $\Gamma(\cdot), \Gamma(\cdot, \cdot)$ are the gamma and incomplete gamma fuctions defined in [10, eq. (8.310.1)] and [10, eq. (8.350.2], respectively. For the direct channel $b_{0}$ we defined $w_{0}=0$, since no extra energy is consumed for the direct $S-D$ transmission.

The conditional bit error probability (BEP), conditioned on the SNR $\gamma$, of the DBPSK signal modulation on an AWGN channel equals to

$$
P_{r}(E \mid \gamma)=A \exp (-B \gamma),
$$

where $A, B$ equal to $1 / 2$ and 1 , respectively. Likewise, (17) represents an approximation of the BEP of the $M$-PSK and $M$ QAM signal modulations on an AWGN channel; in such case, $A$ and $B$ are derived by fitting the exact conditional BEP curve to the approximated BEP of (17) (see [11], [8]). For instance, for the BPSK case we found via numerical evaluations that $A$ and $B$ are approximately equal to 0.2568 and 1.2 respectively, when $\gamma$ lies in the interval $[0 \mathrm{~dB}, 20 \mathrm{~dB}]$.

Lemma 1: The coefficients (profits) in the traditional knapsack problem (eq. (4)) that minimize the ABEP for the DBPSK, $M$-PSK and $M$-QAM signal modulations are

$$
p_{j}=\log _{\beta}\left[\frac{1}{\mathcal{M}_{\gamma_{j}}(-B)}\right], j=0,1, \ldots, L,
$$

where $\beta>1^{4}$ and $\mathcal{M}_{\gamma_{j}}(s)$ is the moment generating function

\footnotetext{
${ }^{4}$ The base of the logarithm in (18) can be any positive real number in the interval $(1, \infty)$. However, small values close to 1 result in better discreteness among the branches.
} 
(MGF) of $\gamma_{j}$ defined as

$$
\mathcal{M}_{\gamma_{j}}(s) \triangleq E\left[\exp \left(s \gamma_{j}\right)\right]=\int_{0}^{\infty} \exp (s v) f_{\gamma_{j}}(v) d v
$$

where $f_{\gamma_{j}}(\cdot)$ denotes the probability density function of $\gamma_{j}$.

Proof: Please refer to [12].

\section{B. Total Energy Consumption Minimization Under ABEP Constraints}

Similar to (15), the coefficients $p_{j}, j=1, \ldots, L$ in (5) represent the energy consumed by the relay corresponding to the branch $b_{j}$, i.e.,

$$
p_{j}=\left\{\begin{array}{cc}
E\left[\mathcal{E}_{j}\right], & j=1, \ldots, L \\
0, & j=0
\end{array},\right.
$$

where $E\left[\mathcal{E}_{j}\right]$ is given in (16).

Lemma 2: By setting the coefficients $w_{j}$ in the minimization knapsack problem (eq. (5)) as

$$
w_{j}=\log _{\beta}\left[\frac{1}{\mathcal{M}_{\gamma_{j}}(-B)}\right], j=0,1, \ldots, L,
$$

where $\beta>1$, we ensure that the ABEP for the DBPSK, $M$-PSK and $M$-QAM signal modulations does not exceed a predefined threshold $\delta$ (if possible ${ }^{5}$ ).

Proof: Please refer to [12].

\section{NUMERICAL EXAMPLES AND DISCUSSION}

In order to illustrate the performance of the proposed scheme, an extensive set of numerical examples is performed, using the MGF-based approach for the ABEP given in [7, eq. (5.3)], followed by the corresponding simulations. A BPSK modulation scheme is used, and the fading on the $S-D$ and on each $S-R_{j}$ and $R_{j}-D$ channel is considered to be independent and Nakagami- $m$ distributed, with the fading parameter $m$ being a random variable (RV) uniformly distributed in the interval $[1,2.5]$. The average values of the fading attenuation on the direct $S-D$, and each $S-R_{j}$ and $R_{j}-D$ channel are considered continuous independent and identically distributed (i.i.d.) lognormal RVs, with mean and standard deviation 0.25 and 0.1 , respectively, for the $S-D$, and 0.5 and 0.2 , respectively, for the $S-R_{j}$ and $R_{j}-D$ channels. Also, for our example $N_{0}=0.1$, and the power transmitted by the source node $S$ is normalized to unity; for this reason, in the following the term normalized will denote normalization with respect to the average power transmitted by $S$.

The main advantage of the traditional and the minimization knapsack problem utilization in multi-user cooperative diversity systems is presented in Tables I and II. In these Tables, the proposed model is compared, in terms of ABEP and normalized total power consumption, with two different longterm selection schemes: a) the conventional one, involving

\footnotetext{
${ }^{5}$ It is evident that Lemma 2 does not hold when the activation of all the available relays leads to an ABEP which is greater than the predefined threshold $\delta$.
}

TABLE I

LTNS TRADITIONAL KNAPSACK PROBLEM: ABEP AND NoRMALIZED TOTAL POWER CONSUMPTION

\begin{tabular}{|c|c|c|c|c|}
\hline \hline \multicolumn{5}{|c|}{ ABEP / Consumption } \\
\hline \hline \multirow{3}{*}{$C_{\max }=1.6$} & $\begin{array}{c}\text { Avail. } \\
\text { Users }\end{array}$ & Knapsack & Highest Eff. & All Users \\
\cline { 2 - 6 } & 5 & $1.3 E^{-3} / 1.52$ & $8.4 E^{-2} / 0.26$ & $5.2 E^{-4} / 1.96$ \\
\cline { 2 - 6 } & 10 & $6.2 E^{-4} / 1.60$ & $8.4 E^{-2} / 0.26$ & $1.6 E^{-5} / 3.91$ \\
\cline { 2 - 6 } & 15 & $4.1 E^{-4} / 1.40$ & $7.5 E^{-2} / 0.20$ & $1.5 E^{-7} / 6.19$ \\
\cline { 2 - 6 }$C_{\max }=6$ & 20 & $2.8 E^{-4} / 1.56$ & $7.5 E^{-2} / 0.20$ & $2.2 E^{-9} / 7.90$ \\
\cline { 2 - 6 } & 5 & $5.2 E^{-4} / 1.96$ & $8.4 E^{-2} / 0.26$ & $5.2 E^{-4} / 1.96$ \\
\cline { 2 - 6 } & 10 & $1.6 E^{-5} / 3.90$ & $8.4 E^{-2} / 0.26$ & $1.6 E^{-5} / 3.91$ \\
\cline { 2 - 6 } & 15 & $3.7 E^{-7} / 5.36$ & $7.5 E^{-2} / 0.20$ & $1.5 E^{-7} / 6.19$ \\
\cline { 2 - 6 } & 20 & $3.4 E^{-8} / 5.47$ & $7.5 E^{-2} / 0.20$ & $2.2 E^{-9} / 7.90$ \\
\hline
\end{tabular}

TABLE II

LTNS Minimization KNAPSACK PROBLEM: ABEP AND NORMALIZED TOTAL POWER CONSUMPTION

\begin{tabular}{||c|c|c|c|c|}
\hline \hline \multicolumn{5}{|c|}{ ABEP / Consumption } \\
\hline \multirow{3}{*}{$\delta=10^{-4}$} & $\begin{array}{c}\text { Avail. } \\
\text { Users }\end{array}$ & Knapsack & Highest Eff. & All Users \\
\cline { 2 - 6 } & 5 & $5.2 E^{-4} / 1.96$ & $9.7 E^{-2} / 0.29$ & $5.2 E^{-4} / 1.96$ \\
\cline { 2 - 6 } & 10 & $8.3 E^{-5} / 3.11$ & $9.7 E^{-2} / 0.29$ & $1.6 E^{-5} / 3.90$ \\
\cline { 2 - 6 } & 15 & $1.7 E^{-5} / 2.94$ & $8.6 E^{-2} / 0.21$ & $1.5 E^{-7} / 6.19$ \\
\cline { 2 - 6 } & 20 & $2.9 E^{-5} / 2.25$ & $8.6 E^{-2} / 0.21$ & $2.2 E^{-9} / 7.90$ \\
\hline \hline \multirow{5}{*}{$\delta=10^{-6}$} & 5 & $5.2 E^{-4} / 1.96$ & $9.7 E^{-2} / 0.29$ & $5.2 E^{-4} / 1.96$ \\
\cline { 2 - 6 } & 10 & $1.6 E^{-5} / 3.91$ & $9.7 E^{-2} / 0.29$ & $1.6 E^{-5} / 3.90$ \\
\cline { 2 - 6 } & 20 & $1.7 E^{-7} / 4.67$ & $8.6 E^{-2} / 0.21$ & $1.5 E^{-7} / 6.19$ \\
\hline
\end{tabular}

activation of all the available nodes, and b) the scheme where a single branch $b_{\eta}$, the one with the highest efficiency is activated through

$$
\eta=\arg \max _{j=1, \ldots, L} e_{j}
$$

where $e_{j}$ defined in (6) or (7).

In general, the proposed model appears to achieve the optimal compromise between error performance and energy consumption, performing in a way similar to the case when a single or all the available nodes are activated. This is determined by the energy consumption constraint $C_{\max }$, or by the equivalent $\mathrm{ABEP}$ one $\delta$, corresponding to the traditional and the minimization knapsack problem, respectively. If, for example, the value of $C_{\max }$ is small, or the value of $\delta$ is high enough so as only one user is activated, the knapsack model reduces to the scheme where the only cooperating node is 


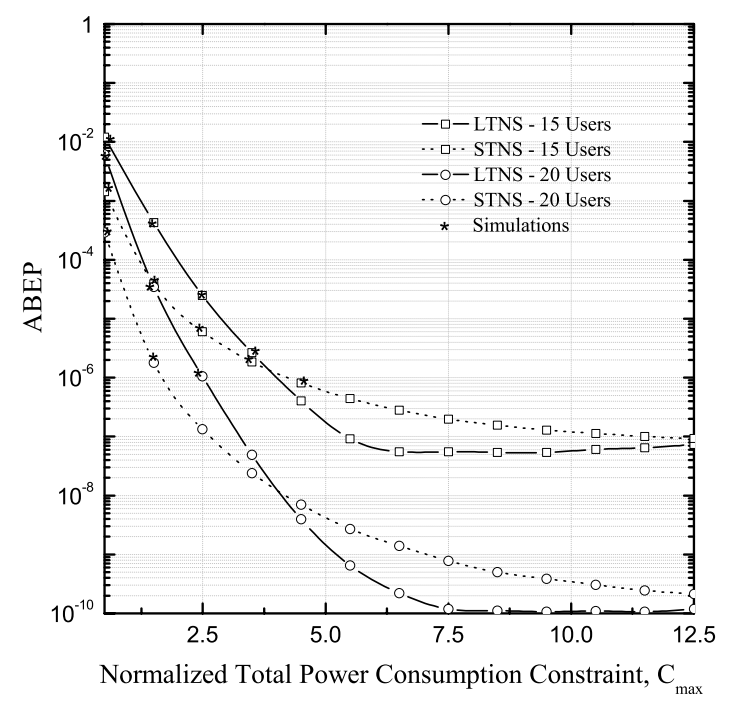

Fig. 1. Traditional Knapsack Problem: ABEP vs Energy Consumption Constraint.

the one with the highest efficiency. Likewise, the knapsack scheme can act as an "all available users" selection model, by setting high or small values for $C_{\max }$ or $\delta$, respectively. Thus, it is evident that the proposed selection method allows the system administrator to easily adapt the system's performanceconsumption tradeoff, according to its needs.

\section{A. ABEP Minimization Under Energy Consumption Con- straints}

Fig. 1 depicts the system's ABEP versus the normalized value of $C_{\max }$. The error performance of the LTNS and STNS is presented, for 15 and 20 available cooperating users. The comparison shows that in the low energy constraint regime STNS outperforms LTNS; however, in the high energy constraint regime, LTNS leads to an error performance which is generally better than the corresponding short-term one, and asymptotically equal to it as the energy consumption constraint grows infinitely large. This can be intuitively explained considering that STNS optimizes the error performance corresponding to each time interval $T_{c}$, under the same constraint with LTNS; this acts as a STNS advantage for small values of $l{ }^{6}$ and as a LTNS advantage as $l$ converges to $L$. Indeed, since the number of possible alternative selections decreases as $l$ approaches $L$, the STNS advantage of optimizing the selection every $T_{c}$ is counterbalanced by its inability of setting a flexible energy constraint, as it was described in the last paragraph of Section IV, resulting finally in worse error performance.

\section{B. Energy Consumption Minimization Under ABEP Con- straints}

Fig. 2 depicts the normalized total energy consumption per unit time versus the ABEP upper bound $\delta$. The energy consumption dependence on $\delta$ presented in this figure is

\footnotetext{
${ }^{6}$ Recall that $l$ represents the number of selected branches during the selection interval.
}

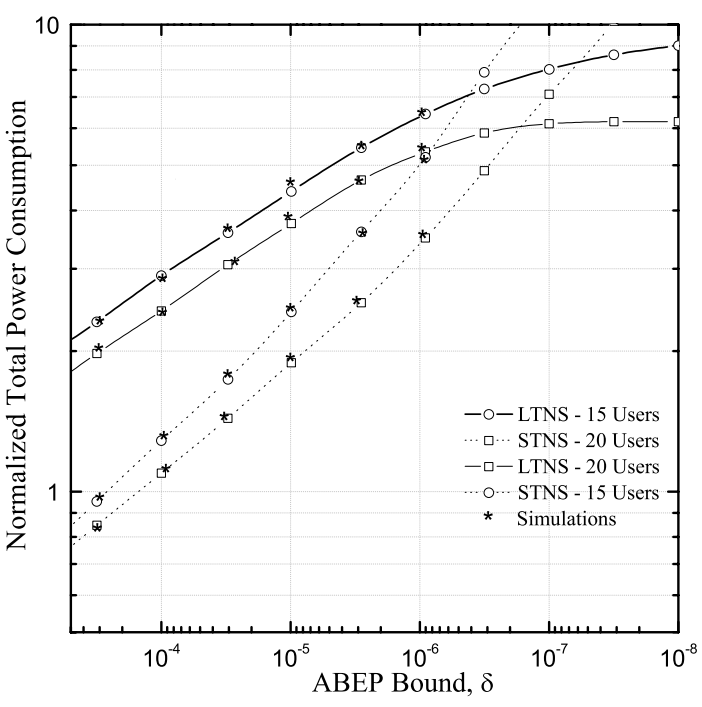

Fig. 2. Minimization Knapsack Problem: Energy Consumption vs ABEP bound.

similar to the ABEP dependence on the energy consumption constraint $C_{\max }$, as it is shown in Fig. 1; in general, large number of possible alternative selections results in better STNS performance and vice versa.

Nevertheless, the difference between STNS and LTNS performance is almost negligible. Hence, considering that LTNS is simpler, in terms of algorithm repetition and protocol complexity, it represents a more efficient solution to the branch selection problem.

\section{REFERENCES}

[1] A. Sendonaris, E. Erkip, and B. Aazhang, "User cooperation diversityPart I: System description," IEEE Trans. Commun., vol. 51, pp. 19271938, Nov. 2003

[2] — "User cooperation diversity-Part II: Implementation aspects and performance analysis," IEEE Trans. Commun., vol. 51, pp. 1927-1938, Nov. 2003.

[3] J. N. Laneman, D. N. C. Tse, and G. W. Wornell, "Cooperative diversity in wireless networks: Efficient protocols and outage behaviour," IEEE Trans. Inform. Theory, vol. 50, pp. 3062-3080, Dec. 2004.

[4] A. Bletsas, A. Khisti, D. P. Reed, and A. Lippman, "A simple cooperative diversity method based on network path selection," IEEE J. Sel. Areas Commun., vol. 24, pp. 659-672, Mar. 2006.

[5] H. Kellerer, U. Pferschy, and D. Pisinger, Knapsack Problems, 1st ed. Berlin: Springer, 2004.

[6] J. N. Laneman, "Cooperative Diversity in Wireless Networks: Algorithms and Architectures," Ph.D. dissertation, Massachusetts Institute of Technology, Cambridge, MA, Aug. 2002.

[7] M. K. Simon and M.-S. Alouini, Digital Communication over Fading Channels, 2nd ed. New York: Wiley, 2005.

[8] Y.-Ko and M.-S. Alouini, "Estimation of Nakagami- $m$ fading channel parameters with application to optimized transmitter diversity systems," IEEE Trans. Wireless Commun., vol. 2, pp. 250-259, Mar. 2003.

[9] R. You, H. Li, and Y. Bar-Ness, "Diversity combining with imperfect channel estimation," IEEE Trans. Commun., vol. 53, pp. 1655-1662, Oct. 2005.

[10] I. S. Gradshteyn and I. M. Ryzhik, Table of Integrals, Series, and Products, 6th ed. New York: Academic, 2000.

[11] K. J. Hole, H. Holm, and G. E. Øien, "Adapted multidimensional coded modulation over flat fading channels," IEEE J. Sel. Areas Commun., vol. 18, pp. 1153-1158, July 2000 .

[12] Internet. [Online]. Available: http://users.auth.gr/geokarag/Reports.htm 